\title{
ELECTROSTATIC FRAGMENTATION OF IRREGULARLY SHAPED PARTICLES
}

\author{
TADASHI MUKAI \\ Dept. of Earth Sciences \\ Faculty of Science, Kobe University \\ Nada, Kobe 657, Japan
}

\begin{abstract}
An enhancement of the electrostatic stress forces due to the charge concentration on a position with small radius of curvature on the surface of irregularly shaped particle causes the fragmentation of fluffy particle more than expected for a spherical particle. This mechanism may act to produce "dust clusters" as detected in comet P/Halley by Simpson et al.(1987) and also "dust swarms" near the Earth as reported in Fechtig(1982).
\end{abstract}

\section{INTRODUCTION}

There are many observed evidence to suggest the destruction of interplanetary dust particles. For example, Simpson et al.(1987) found higher counts of impact particles in smaller grains within short( 1 or 2 seconds) time scale in the coma of comet $\mathrm{P} / \mathrm{Halley}$ ("dust clusters"). They have suggested that the fragmentation of parent dust particles produced these debris in the cometary coma. HEOS 2 experiments revealed an enhancement of dust impact events within a very short time interval. These groups of particles were also interpreted as the debris of large particles(see Fechtig 1982).

Many mechanisms to destroy the particles have been proposed in the previous papers, e.g. evaporation breakup(Mukai 1984), rotational bursting(Misconi 1976), collisional breaknp with high energy particles(Mukai 1980) and electrostatic breakup(Rhee 1976, Boehnhardt and Fechtig 1987). In this short note, we will focus our study on the destruction of irregularly shaped particles caused by electrostatic forces.

\section{MECHANISM}

\subsection{An equilibrium electric potential}

A particle attains an equilibrinm electric potential on its surface in the interplanetary environment. Several charging processes act on the grain(see, e.g. Mukai 1981, Boehhardt and Fechtig 1987). Photoelectric emission caused by sunlight mainly leads a positive state

A.C. Levasseur-Regourd and H. Hasegawa (eds.), Origin and Evolution of Interplanetary Dust, 371-374.

(C) 1991 Kluwer Academic Publishers, Printed in Japan. 
of electric potential on the grain surface, and sticking of electrons leads its negative state. Field emission of electron prevents a grain from getting huge negative charges especially on small grains.

For example, the equilibrium potential for a graphite grain in the day side of the earth's magnetosphere is derived from a comparison of emission/sticking rates of charges as shown in figure 1. In this figure, a cross point between photoelectric emission rate (solid curve) and electron sticking rate(dashed curves) indicates the equilibrium potential U. A number density of electrons is assumed $0.1 \mathrm{~cm}^{-3}$. Other physical parameters of interest are referred in Mukai(1981). We can estimate from figure 1 that the electric potential $U$ of graphite grain in the day side of the earth's magnetosphere becomes roughly $+(5 \sim 8)$ volts. By using a similar way, the values of $U$ in the night side can be estimated, i.e. $U=(-12 \sim+4)$ volts.

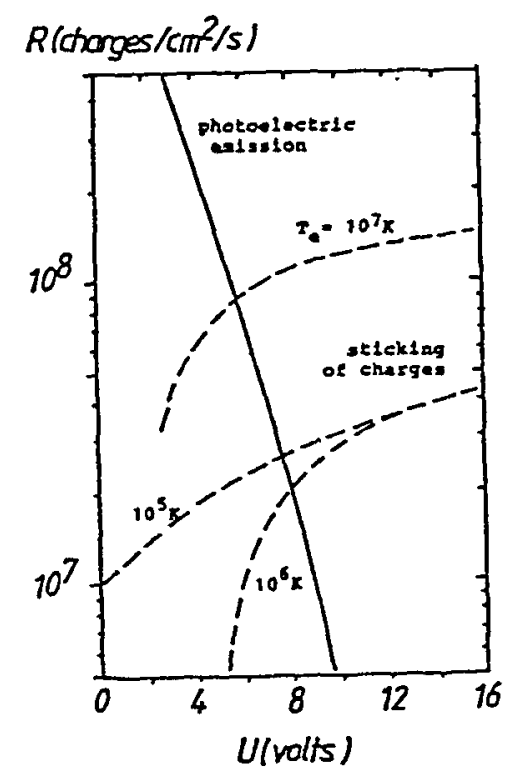

Figure 1. Emission/sticking rates $(R)$ of charges on the surface of graphite grain in the day side of the Earth's magnetosphere. $T_{e}$ denotes the electron temperature.

Boehnhardt and Fechtig(1987) derived the values of $U$ in the coma of comet P/Halley as nearly $+(2 \sim 6)$ volts for carbon particle and $+(7 \sim 11)$ volts for silicate grain. It would be noted that extremely huge values of $U$ are not attained in the interplanetary environment.

\subsection{Fragmentation}

The Maxwell's stress tensor force $F$ is given by $F=\frac{1}{2} \varepsilon_{0} E^{2}$, where $E$ is the strength of electric field on the surface of a grain, and $\varepsilon_{0}$ denotes the dielectric constant of the vacuum.

For a sphere with a radius $r$ and a surface electric potential $U$, the density of surface charges $\sigma_{0}$ is expressed by $\sigma_{0}=Q /\left(4 \pi r^{2}\right)=\varepsilon E=\varepsilon U / r$, where $Q$ means the total charges on the surface of the grain and $\varepsilon$ is the dielectric constant of the grain material. 
It is well known that the charge distribution on the surface of an irregularly shaped particle is not uniform. We assume that the irregularity on the surface of a roughly spherical particle with a radius $r$ is represented by a part of a sphere with a small radius of curvature $\rho$ (see figure 2).
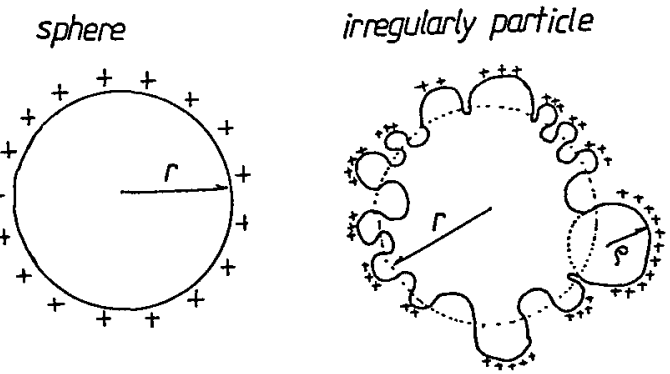

Figure 2. Charge distribution on the surface of a particle

The density of charges $\sigma$ on a position with a radius of curvature $\rho$ is given by

$$
\sigma=\varepsilon \frac{U}{\rho}=\sigma_{\bullet} \frac{r}{\rho}
$$

Consequently, the stress tensor force $F_{i}$ on this position becomes

$$
F_{i}=F\left(\frac{r}{\rho}\right)^{2}
$$

This result implies that the tensor stress force $F_{i}$ acted on the position with a small radius of curvature is significantly stronger than those $F$ expected on the average surface of a sphere.

\section{CONCLUSIONS}

In figure 3, a dashed line shows the tensor stress force $F$ caused by the surface charges of $U=5$ volts as a function of a radius of a spherical particle $r$. Since the tensile strength of real grain material is rather high, we had to assume very fragile material to breakup the grains by the electrostatic fragmentation (see, e.g. Boehnhardt and Fechtig 1987).

When we consider the charge concentration on the surface of an irregularly particle, however, enough stress tensor forces to disrupt the particle can be expected, as shown in figure 3 by the solid curves. This suggests that the interplanetary particle with irregularly shape is broken up by electrostatic forces more than expected for a spherical grain. Until now, our knowledge of the irregularity of the interplanetary dust particles is very poor. Therefore, we need in situ measurements to know the shape of grains in the interplanetary space for studying not only their scattering/thermal emission properties, but their destruction mechanisms. 


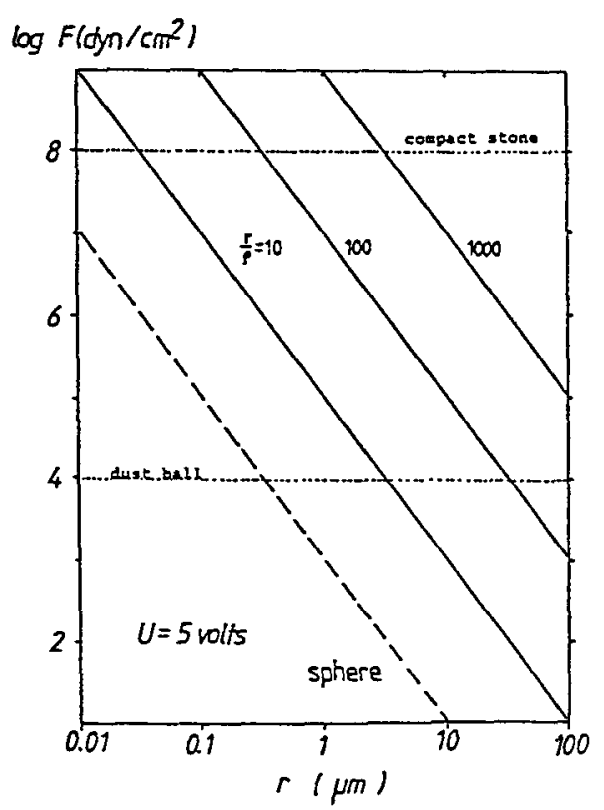

Figure 3. Stress force $F$ vs, a grain radius $r$. A dashed line denotes the electrostatic forces on the surface of a sphere with a radius $r$. The solid lines show the forces on the position with small radius of curvature $\rho$.

Acknowledgement. This work was supported in part by the Scientific Research Fund of the Minister of Education, Science, and Culture(02640205)

\section{References}

Boehnhardt, H. and Fechtig, H.(1987) 'Electrostatic charging and fragmentation of dust near $\mathrm{P} /$ Giacobini-Zinner and $\mathrm{P} / \mathrm{H}$ alley', Astron.Astrophys. 187, 824-828.

Fechtig, H.(1982) 'Cometary dust in the solar system', "Comets"(ed.L.L.Wilkening, The Univ. of Arizona Press), 370-382.

Misconi, N.Y.(1976) 'On the rotational bursting of interplanetary dust particles', Geophys. Res. Letters 3, 585-588.

Mukai, T.(1980) 'Grain disruption by collisions with solar energetic particles', "Solid Particles in the Solar System" (eds. I.Halliday and B.A.McIntosh, D.Reidel Publ. Co.) 385-389.

Mnkai, T.(1981) 'On the charge distribution of interplanetary grains', Astron. Astrophys. $99,1-6$.

Mukai, T.(1984) 'Heterogeneous grain destruction near the sun', Earth, Moon, Planets 30, 99-103.

Rhee, J.W.(1976) 'Electrostatic disruption of lunar dust particles', Lecture Notes in Phys. 48, 238-240.

Simpson, J.A., Rabinowitz, D., Tuzzolino, A.J., Ksanfomality, L.V. and Sagdeev, R.Z.(1987) 'The dust coma of comet $\mathrm{P} / \mathrm{Halley}$ : measurements on the Vega-1 and Vega-2 spacecraft', Astron. Astrophys. 187, 742-752. 